\title{
Stage IIB Bone Cancer AJCC v8
}

National Cancer Institute

\section{Source}

National Cancer Institute. Stage IIB Bone Cancer AJCC v8. NCI Thesaurus. Code C136618.

Stage IIB includes: T2, NO, M0, G2 or G3. T2: Tumor measuring more than $8 \mathrm{~cm}$ in greatest dimension. NO: No regional lymph node metastasis. M0: No distant metastasis. G2: Moderately differentiated, high grade. G3: Poorly differentiated, high grade. (AJCC 8th ed.) 